\title{
Steady-State Levels of Troponin and Brain Natriuretic Peptide for Prediction of Long-Term Outcome after Acute Heart Failure with or without Stage 3 to 4 Chronic Kidney Disease
}

\author{
Yutaka Endo ${ }^{1 *}$, Shun Kohsaka ${ }^{1}$, Toshiyuki Nagai ${ }^{1}$, Kimi Koide ${ }^{1}$, \\ Masashi Takahashi ${ }^{1}$, Yuji Nagatomo ${ }^{1}$, Kazuki Oshima $^{1}$, Hiroaki Miyata ${ }^{2}$, \\ Keiichi Fukuda ${ }^{1}$ and Tsutomu Yoshikawa ${ }^{3}$ \\ ${ }^{1}$ Division of Cardiology, Keio University School of Medicine, Tokyo, Japan \\ ${ }^{2}$ Department of Healthcare Quality Assessment, Graduate School of Medicine, \\ University of Tokyo, Tokyo, Japan \\ ${ }^{3}$ Director, Division of Cardiology, Sakakibara Heart Institute, Tokyo, Japan.
}

\begin{abstract}
Authors' contributions
This work was carried out in collaboration between all authors. YE collected the clinical data, designed the study, performed the statistical analysis, wrote the protocol, wrote the first draft

of the manuscript, and managed the literature searches. SK designed the study, wrote the protocol, managed the literature searches and supervised the literature. TN, KK, MT and $Y N$ managed the literature searches. KO collected the clinical data. HM managed the literature searches and performed the statistical analysis. KF managed the literature searches and supervised the literature. TY designed the study, managed the literature searches and supervised the literature. All authors read and approved the final manuscript.
\end{abstract}

Research Article

Received $28^{\text {th }}$ March 2012

Accepted $21^{\text {st }}$ May 2012

Published 23 ${ }^{\text {rd }}$ August 2012

\section{ABSTRACT}

Aim: To determine whether assessment of a combination of steady-state discharge levels of biomarkers improves risk stratification after acute decompensate HF.

Study Design: Retrospective cohort study.

Place and Duration of Study: Keio University Hospital, between January 2006 and September 2011.

We analyzed 244 patients with acute HF due to ischemic or dilated cardiomyopathy who 
were enrolled in a prospective, single institution-based registry between January 2006 and September 2011. Patients were stratified by discharge values of BNP and/or TnT. The primary endpoint was a composite of HF readmission or death during the 2-year period after discharge.

Results: The population was predominantly male $(69.3 \%)$, and the mean age was $66.6 \pm 15.3$ years. Patients with higher BNP levels or detectable TnT had a worse prognosis (BNP45.0\% vs. 18.8\%, $p<0.001$; TnT $43.8 \%$ vs. $25.1 \%, p=0.002$, respectively). The primary event rate was additively worse among patients with both increased BNP levels and detectable TnT compared to those with increased levels of BNP or detectable TnT alone (log-rank $p<0.001$ ). A similar trend was observed in the subgroup of patients with CKD stage III-V $(n=172)$.

Conclusion: Assessment of both BNP and TnT values may have a significant predictive value for HF prognosis, even among patients with CKD, a condition affecting biomarker levels.

Keywords: Biomarkers; heart failure; chronic kidney disease.

\section{INTRODUCTION}

Heart failure (HF) is a disorder that has major clinical and public health impacts worldwide (Young, 2004). In Japan, the number of HF admissions reached 582,000 in 2008 and HF consumes a significant proportion of the funds allocated for cardiovascular care, which totaled $\$ 713$ billion in 2009 (Report from Japanese Health and Welfare, 2009). HF is estimated to affect over 5 million people in the US, and it is the most common Medicare diagnosis-related group. Furthermore, as the population ages, HF prevalence increases; currently, the HF incidence approaches 10 per 1000 among those older than 65 years, and the estimated cost of HF treatment in the United States was \$37 billion in 2009 (Lloyd-Jones et al., 2009).

Several major advances in the management of HF have been achieved in the past decade, and some data suggest that these new advances are beginning to impact the prognosis of HF in the community (Polanczyk et al., 2000). One such advance is the reorganization of high-risk patients stratified by biomarkers. In particular, biomarkers representing a patient's fluid status (e.g., brain natriuretic peptide [BNP]) or degree of myocardial injury (e.g., highly sensitive troponins [TnT]) are valuable tools for predicting the long-term prognosis of $\mathrm{HF}$ (Peacock et al., 2008; Braunwald, 2008; Brugger-Andersen et al., 2008; Felker et al., 2000).

However, the impact of the combination of BNP and TnT values has not been thoroughly investigated, particularly among patients with chronic kidney disease (CKD). Kidney disease affects HF through various mechanisms, including those described by the low-flow-state hypothesis, intraabdominal and central venous pressure elevation, sympathetic overactivity, rennin-angiotensin-aldosterone system overactivity, and oxidative injury. HF affects biomarker levels, but its impact on the long-term outcome is unclear. Data on biomarker levels are also particularly sparse in the Japanese population where a non-ischemic etiology is the dominant characteristic of HF. Therefore, we sought to investigate whether evaluation of a combination of biomarkers could improve risk stratification of patients with acute decompensated HF, particularly those with CKD. 


\section{MATERIALS AND METHODS}

\subsection{Study Subjects}

Between January 2006 and September 2011, we prospectively registered 244 consecutive patients who were admitted to Keio University Hospital for the treatment of decompensate HF defined according to the Framingham criteria. To avoid the influence of acute coronary events and renal dysfunction, patients with clinical or electrocardiographic evidence of acute coronary syndrome in the previous 3 months, those with coronary revascularization in the previous 3 months, those with renal failure (serum creatinine concentration, $\geq 2.5 \mathrm{mg} / \mathrm{dl}$ ), and those undergoing hemodialysis were excluded (Srisawasdi et al., 2010). Patients with terminal cancer, infections and inflammatory diseases were also excluded. The primary endpoint was a composite of all-cause mortality plus HF requiring hospitalization. The mean length of follow-up was 730 days (interquartile range 397 to 730 ). Informed consent was obtained from each patient upon enrollment.

Dilated cardiomyopathy is characterized by dilatation and impaired contraction of 1 or both ventricles and an EF of $<45 \%$ without the presence of obstructive coronary disease. Ischemic cardiomyopathy was defined by a-number-of-diseased-vessels classification which is the presence of obstruction (more than $75 \%$ ) in one or more coronary (Felker et al., 2002). The study protocol conformed to the ethical guidelines of the 1975 Declaration of Helsinki, and the study was approved by the institution's human research committee.

\subsection{Measurement of Cardiac Biomarkers}

Plasma TnT and BNP levels were measured before discharge. Commercially available assay kits were used for the measurement of TnT (4th generation high sensitivity assay, Roche Diagnostics, Tokyo, Japan) and BNP (Shionogi, Tokyo, Japan). The lower limit of detection for TnT was $0.01 \mathrm{ng} / \mathrm{mL}$, and BNP was subclassified by level according to receiver operating characteristic (ROC) analysis (Cut-off value; $239.5 \mathrm{pg} / \mathrm{mL}$ ). Serum creatinine and hemoglobin levels were determined by standard laboratory methods. Glomerular filtration rate (GFR) was estimated using the equation from the Modification of Diet in Renal Disease Study: eGFR (mL•min-1•1.73 m-2) =0.741 $\times 175 \times$ Age-0.203 $\times$ SCr$1.154(\times 0.724$ for females). CKD was defined as an eGFR of $<60 \mathrm{~mL} / \mathrm{min}$ at the time of discharge. Diabetes mellitus was defined according to the criteria of the American Diabetes Association. Before discharge, experienced technicians who had no knowledge of the biochemical data performed two-dimensional echocardiography in a standard manner using a Hewlett Packard 5500. Clinical data were obtained by interviewing patients and from hospital medical records. Physicians were blinded to the data on biochemical markers and treatment was selected based on the patient's symptoms and physical findings.

\subsection{Statistical Analysis}

Categorical variables were expressed as numbers (percentages) and continuous variables were expressed as the mean \pm standard deviation. An unpaired t-test and chi-square test were used for between-group comparisons of continuous and categorical variables, respectively. If the data were skewed, the nonparametric Mann-Whitney test was used to compare continuous variables. ROC analysis was performed to determine the cut-off values for the conversion of continuous variables into categorical variables when analyzing BNP. Overall survival and survival without hospitalization for HF were analyzed by the Kaplan- 
Meier method, and the curves were compared by the log-rank test. We also performed an analysis based on the presence/absence of CKD.

Univariate and multivariate Cox regression analyses were performed to determine the associations between TnT and other variables. For each covariate, categorical variables were allowed to enter in a stepwise forward multivariate Cox model with the use of a probability value $\leq 0.10$ for inclusion or $\leq 0.05$ for deletion. The multivariate model included categorical variables that were statistically significant according to univariate analysis as well as clinically important. A P value of $<0.05$ was considered significant. Statistical analyses were performed with SPSS version 16.0 software (SPSS Inc., Chicago, Illinois).

\section{RESULTS AND DISCUSSION}

\subsection{Study Population}

At baseline, the mean age of all patients was $66.6 \pm 15.3$ years; $69.3 \%$ were men. About half of the patients had non-ischemic cardiomyopathy (47.5\%). TnT was detectable $(\geq 0.01 \mathrm{ng})$ in $73(29.9 \%)$ patients at discharge, with interquartile range 0.00 to 0.02 . The median level of BNP was $206 \mathrm{pg} / \mathrm{mL}$ (interquartile range 108 to 490) at discharge. Notably, our population included a large group of patients with CKD (68.0\%). Threshold for BNP was determined via ROC curve analysis $(239.5 \mathrm{pg} / \mathrm{mL})$. TnT threshold for detectable vs non detectable was 0.01 $\mathrm{ng} / \mathrm{mL}$.

The patients were subsequently divided into 3 groups based on biomarker levels at discharge: patients with both lower BNP levels and undetectable TnT (group 0, $n=107$ ); those with either higher BNP levels or detectable TnT (group 1, $n=84$ ); and those with both higher BNP level and detectable TnT (group 2, $n=53$ ). Patients with elevated biomarker levels also had a greater likelihood of diabetes mellitus $(p=0.007)$, factors suggestive of impaired renal function (such as eGFR, $p<0.001)$, elevated blood urea nitrogen $(p<0.001)$, and a higher rate of CKD $(p<0.001)$ and anemia $(p<0.001)$ at discharge $($ Table 1$)$.

\subsection{Outcomes}

During the 2-year follow-up period, 75(30.7\%) events were recorded (28 deaths and 66 readmissions). Kaplan-Meier event curves comparing the prognosis of patients with higher vs. lower BNP levels, and patients with detectable vs. undetectable TnT levels are shown in Fig. 1A. During the follow-up period, primary event rates were higher among patients with elevated BNP levels (log-rank test; $p<0.001)$ or detectable TnT $(p=0.007)$ compared with those with lower BNP levels or undetectable TnT. When the patients were stratified into 3 groups based on biomarker values, the primary event rate was additively worse among patients with both increased BNP values and detectable TnT levels (Fig. 1B; log-rank $\mathrm{p}<0.001)$. For the individual comparison, there were statistically significant differences in the outcome of both biomarker positive groups (double and single positive group) compared to control group $(p<0.001)$. There were no statistically significant difference in the outcome of double-positive $(\mathrm{TNT}[+] / \mathrm{BNP}[+])$ when compared to single-positive group $(\mathrm{TNT}[+]$ or BNP[+]; $\mathrm{p}=0.318$ ). Similar trend was seen when the outcome of death and heart failure were assessed individually. 
Table 1. Baseline characteristics of the patients by the BNP and TnT values

\begin{tabular}{|c|c|c|c|c|c|c|}
\hline & & All (=244) & $\begin{array}{l}\text { Group } \\
0(=107)\end{array}$ & $\begin{array}{l}\text { Group } \\
1(=84)\end{array}$ & $\begin{array}{l}\text { Group } \\
2(=53)\end{array}$ & $\begin{array}{l}P \\
\text { value }\end{array}$ \\
\hline \multicolumn{7}{|c|}{ Patients characteristics } \\
\hline Age & & $66.6 \pm 15.3$ & $61.1 \pm 14.9$ & $69.7 \pm 15.0$ & $72.9 \pm 12.8$ & $<0.001$ \\
\hline Male (\%) & & 69.3 & 70.1 & 66.7 & 71.7 & 0.799 \\
\hline \multirow[t]{3}{*}{ Etiology (\%) } & DCM & 47.5 & 58.9 & 36.9 & 41.5 & 0.018 \\
\hline & $\mathrm{ICM}$ & 27.0 & 18.7 & 32.1 & 35.8 & \\
\hline & others & 25.4 & 22.4 & 31.0 & 22.6 & \\
\hline DM (\%) & & 30.5 & 24.3 & 27.4 & 48.1 & 0.007 \\
\hline AF/Af (\%) & & 34.0 & 29.0 & 41.7 & 32.1 & 0.174 \\
\hline smoke (\%) & & 36.1 & 42.7 & 34.4 & 25.0 & 0.153 \\
\hline BMI & & $23.3 \pm 5.6$ & $24.3 \pm 4.7$ & $22.2 \pm 6.3$ & $22.3 \pm 6.1$ & 0.030 \\
\hline HTN (\%) & & 44.6 & 44.0 & 43.8 & 47.2 & 0.914 \\
\hline \multicolumn{7}{|l|}{ On admission } \\
\hline NYHA $2(\%)$ & & 77.2 & 70.2 & 82.1 & 83.0 & 0.079 \\
\hline eGFR & & $50.8 \pm 20.2$ & $58.4 \pm 17.1$ & $48.8 \pm 20.9$ & $38.6 \pm 18.1$ & $<0.001$ \\
\hline CKD (\%) & & 68.0 & 52.3 & 76.2 & 86.8 & $<0.001$ \\
\hline $\mathrm{Hb}$ & & $12.9 \pm 2.4$ & $13.6 \pm 2.2$ & $12.7 \pm 2.4$ & $11.7 \pm 2.3$ & $<0.001$ \\
\hline sBP & & $130.0 \pm 29.2$ & $131.4 \pm 28.3$ & $128.8 \pm 30.5$ & $129.1 \pm 29.2$ & 0.808 \\
\hline $\mathrm{HR}$ & & $89.1 \pm 24.9$ & $90.6 \pm 27.8$ & $86.8 \pm 23.6$ & $89.6 \pm 20.2$ & 0.602 \\
\hline $\mathrm{Na}$ & & $139.5 \pm 4.2$ & $140.5 \pm 3.1$ & $138.9 \pm 5.0$ & $138.6 \pm 4.3$ & 0.007 \\
\hline K & & $4.4 \pm 0.5$ & $4.3 \pm 0.4$ & $4.4 \pm 0.5$ & $4.5 \pm 0.7$ & 0.126 \\
\hline \multicolumn{7}{|l|}{ On discharge } \\
\hline \multirow{2}{*}{\multicolumn{2}{|c|}{$\begin{array}{l}\text { NYHA =>2 } \\
(\%) \\
\text { LAD }\end{array}$}} & 2.1 & 0 & 2.4 & 5.8 & 0.055 \\
\hline & & $45.1 \pm 10.2$ & $45.3 \pm 10.3$ & $43.8 \pm 9.3$ & $46.6 \pm 11.7$ & 0.324 \\
\hline \multicolumn{2}{|l|}{ LVDd } & $56.8 \pm 14.9$ & $58.4 \pm 15.5$ & $54.7 \pm 14.8$ & $56.6 \pm 13.3$ & 0.233 \\
\hline \multicolumn{2}{|l|}{ LVEF } & $35.8 \pm 13.5$ & $35.9 \pm 13.2$ & $35.9 \pm 14.1$ & $35.6 \pm 13.5$ & 0.991 \\
\hline \multicolumn{2}{|l|}{ eGFR } & $50.3 \pm 20.0$ & $57.6 \pm 18.2$ & $47.4 \pm 20.3$ & $40.1 \pm 17.5$ & $<0.001$ \\
\hline \multirow{2}{*}{\multicolumn{2}{|c|}{$\begin{array}{l}\text { CKD (\%) } \\
\mathrm{Hb}\end{array}$}} & 70.5 & 56.1 & 78.6 & 86.8 & $<0.001$ \\
\hline & & $13.0 \pm 2.4$ & $13.9 \pm 2.2$ & $12.8 \pm 2.5$ & $11.8 \pm 2.2$ & $<0.001$ \\
\hline \multicolumn{2}{|l|}{ sBP } & $108.0 \pm 17.4$ & $107.6 \pm 17.2$ & $109.3 \pm 19.0$ & $109.8 \pm 15.0$ & 0.688 \\
\hline \multicolumn{2}{|l|}{$\mathrm{HR}$} & $73.3 \pm 15.4$ & $72.3 \pm 13.6$ & $74.8 \pm 17.4$ & $72.8 \pm 16.0$ & 0.560 \\
\hline \multicolumn{2}{|l|}{$\mathrm{Na}$} & $138.3 \pm 3.7$ & $138.6 \pm 2.9$ & $138.5 \pm 4.1$ & $137.4 \pm 4.4$ & 0.110 \\
\hline \multicolumn{2}{|l|}{$\mathrm{K}$} & $4.5 \pm 0.5$ & $4.5 \pm 0.4$ & $4.4 \pm 0.4$ & $4.6 \pm 0.5$ & 0.036 \\
\hline \multicolumn{2}{|l|}{ BUN } & $25.5 \pm 12.8$ & $21.1 \pm 9.6$ & $25.1 \pm 10.8$ & $35.1 \pm 16.2$ & $<0.001$ \\
\hline \multicolumn{7}{|l|}{ Medication } \\
\hline \multicolumn{2}{|l|}{ BB (\%) } & 78.7 & 82.1 & 75.9 & 76.0 & 0.516 \\
\hline \multicolumn{2}{|l|}{ AceiARB (\%) } & 74.9 & 82.2 & 72.3 & 64.2 & 0.030 \\
\hline \multicolumn{2}{|l|}{ Diuretics (\%) } & 77.9 & 78.5 & 72.6 & 84.9 & 0.236 \\
\hline \multicolumn{2}{|l|}{ Warfarin (\%) } & 37.3 & 38.3 & 34.5 & 39.6 & 0.800 \\
\hline \multicolumn{2}{|l|}{ Aspirin (\%) } & 34.2 & 30.8 & 40.5 & 30.8 & 0.320 \\
\hline \multicolumn{7}{|l|}{ Biomarker } \\
\hline \multirow{2}{*}{\multicolumn{2}{|c|}{$\begin{array}{l}\text { BNP } \\
\text { TnT }\end{array}$}} & $350.3 \pm 439.5$ & $122.8 \pm 94.2$ & $385.7 \pm 267.5$ & $753.6 \pm 704.2$ & $<0.001$ \\
\hline & & $0.03 \pm 0.14$ & 0 & $0.015 \pm 0.039$ & $0.13 \pm 0.28$ & $<0.001$ \\
\hline
\end{tabular}




\subsection{Outcomes of the CKD Subgroup}

The presence of CKD had a significant negative impact on patient survival; overall, CKD patients had significantly more primary events than did non-CKD patients (log-rank test; $p=0.004)$. A separate analysis was performed to demonstrate the impact of biomarkers in CKD patients (Fig. 2A) in comparison to non-CKD patients (Fig. 2B). Similar to our main results, primary events were more frequent among patients with higher BNP levels (log-rank test; $p=0.007)$ or detectable TnT levels $(p=0.082)$ compared with their counterparts. Event rates were also additively worse as the number of elevated biomarkers increased (Fig. 2B; log-rank $p=0.012$ ).

(A)
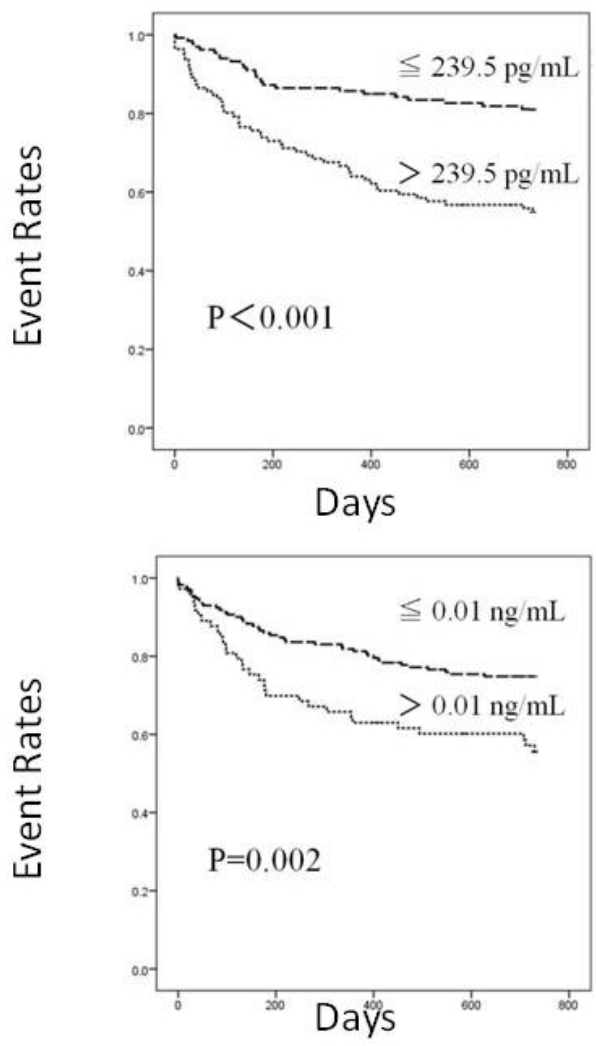

(B)

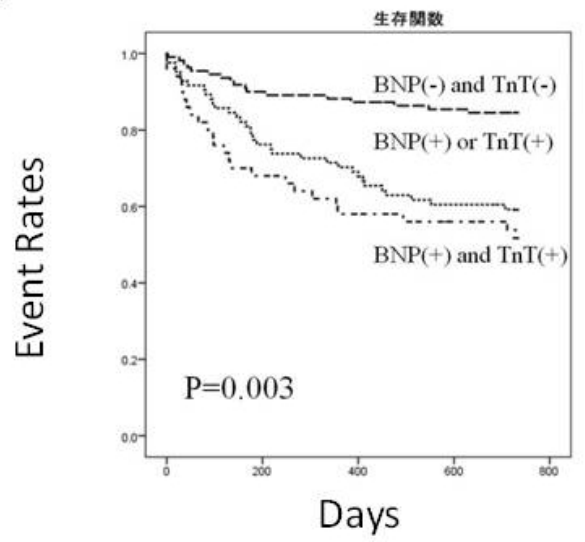

Fig. 1. The long-term prognosis by the stratification of BNP and TnT in all patients. (A) The long-term prognosis of the HF patients stratified by the combination of BNP and TnT level.(B) 
(A)
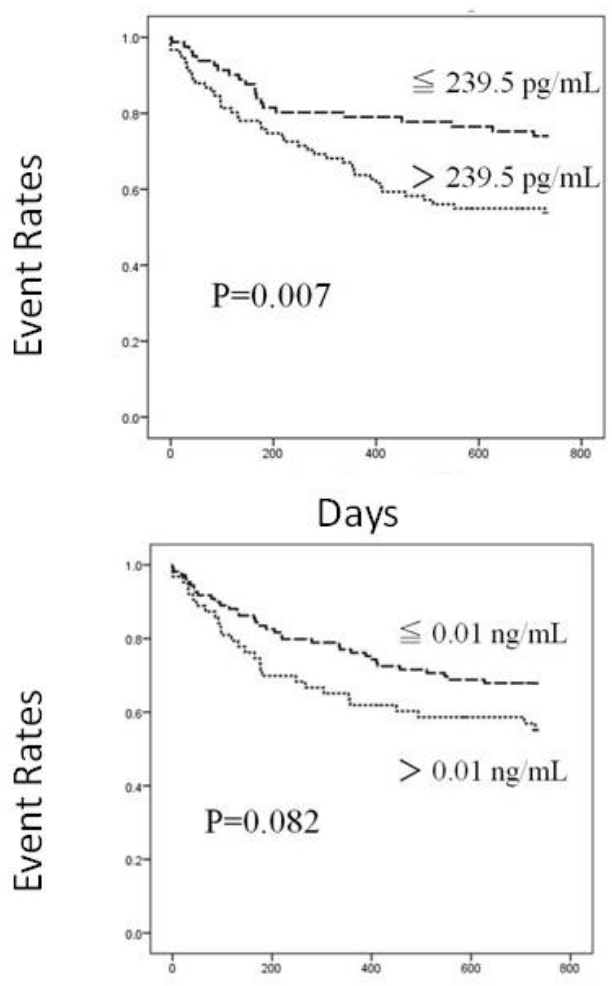

Days
(B)

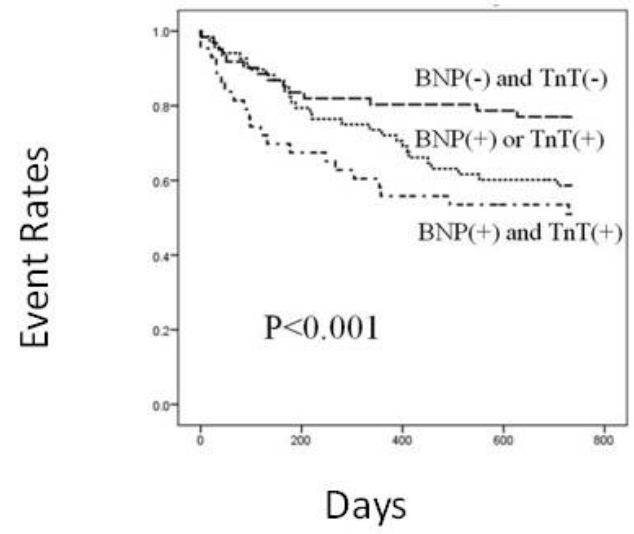

Fig. 2. The long-term prognosis by the stratification of BNP and TnT in subgroup of patients withCKD. (A) The long-term prognosis of the HF patients with CKD stratified by the combination of BNP and TnT level. (B)

\subsection{Univariate and Multivariate Cox Hazard Models}

The results of the univariate and multivariate models are shown in Table 2. Significant predictors of events were age (HR 1.02; 95\% Cl, 1.01-1.04; $p=0.005)$, BNP levels (HR, 2.86; 95\% Cl, 1.77-4.63; $p<0.001$ ), TnT levels ( $\mathrm{HR}, 2.01 ; 95 \% \mathrm{Cl}, 1.27-3.16 ; \mathrm{p}=0.003)$, and the combination of BNP/TnT levels (HR, 1.94; 95\% Cl, 1.46-2.59; $\mathrm{p}<0.001)$. When adjusted for known predictors, the combination of BNP/TnT levels was associated with the combined outcome of HF readmission and all-cause mortality (HR, 1.02;95\% Cl, $1.00-1.05 ; p=0.046$ and $\mathrm{HR}=1.544 ; 95 \% \mathrm{Cl}, 1.11-2.15 ; \mathrm{p}=0.010$, respectively). A similar trend was observed in the analysis of HF patients with CKD (Table 2). 
Table 2. Result of multivariable analysis. Predictors of composite outcome in all patients and in subgroup of patients with CKD

\begin{tabular}{lllll}
\hline All & HR & $\mathbf{9 5 \%} \mathbf{C l}$ & $\mathbf{9 5 \%} \mathbf{C l}$ & $\mathbf{P}$ value \\
\hline Age & 1.003 & 0.982 & 1.023 & 0.806 \\
Left atrial diameter & 1.024 & 1.000 & 1.049 & 0.046 \\
CKD & 1.507 & 0.748 & 3.034 & 0.251 \\
Hemoglobin & 0.957 & 0.848 & 1.079 & 0.473 \\
BUN & 1.007 & 0.986 & 1.029 & 0.504 \\
Use of ACE inhibitors or ARB & 0.680 & 0.398 & 1.163 & 0.159 \\
Positive BNP or TnT values on discharge & 1.544 & 1.110 & 2.146 & 0.010 \\
CKD & & & & \\
& HR & $\mathbf{9 5 \%} \mathbf{C l}$ & $\mathbf{9 5 \%} \mathbf{C l}$ & $\mathbf{P}$ value \\
Left atrial diameter & 1.025 & 0.998 & 1.053 & 0.071 \\
Left ventricular diastolic dimension & 1.025 & 1.000 & 1.051 & 0.048 \\
BUN & 1.014 & 0.992 & 1.036 & 0.224 \\
Use of ACE inhibitors or ARB & 0.595 & 0.324 & 1.023 & 0.063 \\
Positive BNP or TnT values on discharge & 1.435 & 1.020 & 2.020 & 0.008 \\
\hline
\end{tabular}

\subsection{Discussion}

The present study revealed that discharge levels of both BNP and TnT were associated with the composite of $\mathrm{HF}$ readmission and all-cause mortality in patients with acute decompensate HF. In addition, the combination of elevated BNP and TnT levels further stratified the risk and had an additive effect on the patients' long-term prognosis. Measuring the combination of these biomarkers on discharge appeared to be a useful method for stratification of HF patients, including those with CKD.

Ample evidence has demonstrated the value of natriuretic peptides for predicting adverse outcomes, and the prognostic potential of BNP values was examined in the multicenter Rapid Emergency Department Heart Failure Outpatients Trail (REDHOT), which showed that BNP values were very strong predictors of 90-day outcomes (Maisel et al., 2004). The many mechanisms of BNP release in acute HF, including myocyte stretch and cardiac remodeling, show why these markers are so profoundly prognostic in HF patients.

Serum biomarkers of cardiac stress and malfunction as well as myocyte injury have grown in clinical importance for predicting the prognosis of HF patients. TnT is produced from cardiac myocytes as a consequence of myocardial ischemia. The level of TnT has a significant negative predictive value among HF patients with CKD (Koide et al., 2010). Furthermore, patients with preserved kidney condition may very well be able to tolerate ongoing myocardial injury, whereas patients with CKD are strongly affected by troponin leakage (Kociol et al., 2010).

The assessment of a combination of these conventional biomarkers could potentially improve the risk stratification of acute decompensated HF patients; moreover, kits for such assessments are both inexpensive and widely available. In previous studies, Ishii et al. reported that the combination of cardiac TnT and BNP levels on admission might be highly 
effective for the risk stratification of patients with chronic HF (Ishii et al., 2002), but little information is available on the association of this combination of biomarkers after acute decompensation of HF (Nishio et al., 2007; Taniguchi et al., 2006). In our study, CKD had a significant impact on the long-term outcome, as has previously been reported, and we demonstrated that patients with CKD can also be efficiently stratified by levels of BNP, TnT, and the combination of these 2 biomarkers. Patients with even mild chronic renal insufficiency have significantly increased cardiovascular morbidity and mortality, and chronic renal insufficiency also affects concentrations of biomarkers (Heywood et al., 2007; Ronco et al., 2008; McAlister et al., 2004). We showed, for the first time, that the prognostic value of biomarkers and their combination is not necessarily altered by the presence of renal impairment.

Our study has several limitations. Since we performed a retrospective analysis of registry data, we cannot establish cause and effect. However, the associations are consistent with prior analyses of troponin in patients with acute decompensate HF. Plenty of factors could have affected the results of the biomarker tests. First, we used the results of various biomarker assays for which we defined cutoff points rather than core laboratory results. Second, bias may have been introduced because we were unable to analyze patients with HF in whom troponin was not assessed, and we were unable to determine why physicians obtained, or did not obtain, biomarker measurements. Third, our cohort included mainly Japanese individuals, and there is some difference between Japan and western countries in terms of HF etiology and medication (Oshima et al., 2009). We also did not have information on BNP isoform. However, most of them showed equal predictability when compared to original BNP assay (van Kimmenade et al., 2009) and we believe that the absence of BNP isoform measurements will not alter our main results. Fourth, the $4^{\text {th }}$ generation cTnT assay was used in our study, which is no longer available in most parts of the world and BNP was measured with Shionogi assay which is not used outside of Japan. This BNP assay is known to provide lower values than most of the commercially available BNP assays. Thus, the study has limited generalizability, but we believe that they confirm the principle that discharge values of both markers are suitable for risk stratification. Finally, we excluded the patients undergoing haemodialysis (CKD stage 5) since effect of biomarkers was unclear in these patients. BNP is known to increase exponentially with the stage of renal disease and likely skew the main result of our analysis.

\section{CONCLUSION}

In conclusion, our study suggests that levels of both BNP and TnT have a significant predictive value for the prognosis of HF. Assessment of the combination of these biomarkers, which is both inexpensive and readily available, may provide additional information.

\section{ACKNOWLEDGEMENTS}

This research was supported by funding from Japan Society for the Promotion of Science (No. 23591062).

\section{COMPETING INTERESTS}

Authors have declared that no competing interests exist. 


\section{CONSENT}

All authors declare that 'written informed consent was obtained from the patient for publication of this paper.

\section{ETHICAL APPROVAL}

All authors hereby declare that all human studies have been examined and approved by the appropriate ethics committee and have therefore been performed in accordance with the ethical standards laid down in the 1964 Declaration of Helsinki.

\section{REFERENCES}

Braunwald, E. (2008). Biomarkers in heart failure. N Engl J Med., 358, 2148-59.

Brugger-Andersen, T., Ponitz, V., Staines, H., Pritchard, D., Grundt, H., Nilsen, D.W. (2008). B-type natriuretic peptide is a long-term predictor of all-cause mortality, whereas highsensitive C-reactive protein predicts recurrent short-term troponin $\mathrm{T}$ positive cardiac events in chest pain patients: a prognostic study. BMC Cardiovasc Disord., 8, 34.

Felker, G.M., Thompson, R.E., Hare, J.M. et al. (2000). Underlying causes and long-term survival in patients with initially unexplained cardiomyopathy. $\mathrm{N}$ Engl J Med., 342, 1077-84.

Felker, G.M., Shaw, L.D., O'Connor, C.M. (2002). A standardized definition of ischemic cardiomyopathy for use in clinical research. J Am Coll Cardiol., 39, 210-218.

Heywood, J.T., Fonarow, G.C., Costanzo, M.R., Mathur, V.S., Wigneswaran, J.R., Wynne, J. (2007). High prevalence of renal dysfunction and its impact on outcome in 118,465 patients hospitalized with acute decompensated heart failure: a report from the ADHERE database. J Card Fail., 13, 422-30.

Ishii, J., Nomura, M., Nakamura, Y. et al. (2002). Risk stratification using a combination of cardiac troponin $\mathrm{T}$ and brain natriuretic peptide in patients hospitalized for worsening chronic heart failure. Am J Cardiol., 89, 691-5.

Kociol, R.D., Pang, P.S., Gheorghiade, M., Fonarow, G.C., O'Connor, C.M., Felker, G.M. (2010). Troponin elevation in heart failure prevalence, mechanisms and clinical implications. J Am Coll Cardiol., 56, 1071-8.

Koide, K., Yoshikawa, T., Nagatomo, Y. et al. (2010). Elevated troponin T on discharge predicts poor outcome of decompensated heart failure. Heart and Vessels, 25, 217-22.

Lloyd-Jones, D., Adams, R., Carnethon, M. et al. (2009). Heart disease and stroke statistics-2009 update: a report from the American Heart Association Statistics Committee and Stroke Statistics Subcommittee. Circulation, 119, 480-6.

Maisel, A., Hollander, J.E., Guss, D. et al. (2004). Primary results of the Rapid Emergency Department Heart Failure Outpatient Trial (REDHOT). A multicenter study of B-type natriuretic peptide levels, emergency department decision making, and outcomes in patients presenting with shortness of breath. J Am Coll Cardiol., 44, 1328-33.

McAlister, F.A., Ezekowitz, J., Tonelli, M., Armstrong, P.W. (2004). Renal insufficiency and heart failure: prognostic and therapeutic implications from a prospective cohort study. Circulation, 109, 1004-9.

Nishio, Y., Sato, Y., Taniguchi, R. et al. (2007). Cardiac troponin T vs other biochemical markers in patients with congestive heart failure. Circulation journal: official journal of the Japanese Circulation Society, 71, 631-5. 
Oshima, K., Kohsaka, S., Koide, K., Yoshikawa, T. (2009). Reducing the dose of diuretics for heart failure patients: how low can it go? Cardiology, 114, 89.

Peacock, W.F.T., De Marco, T., Fonarow, G.C. et al. (2008). Cardiac troponin and outcome in acute heart failure. N Engl J Med., 2117-26.

Polanczyk, C.A., Rohde, L.E., Dec, G.W., DiSalvo, T. (2000). Ten-year trends in hospital care for congestive heart failure: improved outcomes and increased use of resources. Arch Intern Med., 160, 325-32.

Report from Japanese Health and Welfare (2009).

Ronco, C., Haapio, M., House, A.A., Anavekar, N., Bellomo, R. (2008). Cardiorenal syndrome. J Am Coll Cardiol., 52, 1527-39.

Srisawadi, P., Vanavanan, S., Charoenpanichkit, C. et al. (2010). The effect of renal dysfunction on BNP, NT-pro BNP, and their ratio. AM J Clin Pathol., 133, 14-23.

Taniguchi, R., Sato, Y., Nishio, Y., Kimura, T., Kita, T. (2006). Measurements of baseline and follow-up concentrations of cardiac troponin- $T$ and brain natriuretic peptide in patients with heart failure from various etiologies. Heart and Vessels, 21, 344-9.

van Kimmenade, R.R., Januzzi, J.L., Jr, Bakker, J.A. et al. (2009). Renal clearance of B-type natriuretic peptide and amino terminal pro-B-type natriuretic peptide a mechanistic study in hypertensive subjects. J Am Coll Cardiol., 53, 884-90.

Young, J.B. (2004). The global epidemiology of heart failure. Med Clin North Am., 88, 113543 , ix.

(c) 2012 Endo et al.; This is an Open Access article distributed under the terms of the Creative Commons Attribution License (http://creativecommons.org/licenses/by/3.0), which permits unrestricted use, distribution, and reproduction in any medium, provided the original work is properly cited. 